\title{
Cryo-EM Reveals Active Site Coordination Within a Multienzyme pre-rRNA Processing Complex
}

\author{
M Pillon ${ }^{1}$, A Hsu ${ }^{1}$, J Krahn ${ }^{1}$, J Williams ${ }^{1}$, K Goslen ${ }^{1}$, M Sobhany ${ }^{1}$, M Borgnia ${ }^{1}$, R Stanley ${ }^{2}$ \\ ${ }^{1}$ National Institutes of Environmental Health Sciences, ${ }^{2}$ NIEHS/NIH \\ monica.pillon@nih.gov
}

Ribosome assembly is a complex process reliant on the coordination of trans-acting enzymes to produce functional ribosomal subunits and secure the translational capacity of the cell. Las 1 is a recently discovered endoribonuclease that assembles into a multienzyme complex with the Grc3 polynucleotide kinase to orchestrate the targeted removal of a transcribed spacer (ITS2) from precursor ribosomal RNA (pre-rRNA). The essential Las1 endoribonuclease cleaves the ITS2 spacer at a defined site to initiate pre-rRNA processing. The Grc3 polynucleotide kinase subsequently phosphorylates the resulting 5'-hydroxyl RNA to signal for 5'- and 3'-exoribonucleases to degrade the ITS2. Disruption of mammalian Las1-Grc3 has been linked to congenital lethal motor neuron disease and X-linked intellectual disability disorders, thus highlighting its importance in human health; yet, its mechanism of action remains unclear. Here we report that the Las1 endoribonuclease assembles into a higher-order tetrameric complex with its binding partner the Grc3 polynucleotide kinase, which is essential for the activation of its nuclease and kinase functions. To understand how Las1-Grc3 achieves its strict nuclease specificity and coordinates its dual enzymes, we determined a series of high-resolution cryo-EM structures of Las1-Grc3 in multiple conformational states. Structural characterization of Las1-Grc3 reveals its molecular architecture harboring a composite nuclease active site flanked by two discrete RNA kinase sites. Coupled with functional studies, we identify molecular features crucial for RNA specificity and two molecular switches that coordinate nuclease and kinase function. Together, our structures and corresponding functional studies establish how Las1-Grc3 couples its enzymatic functions to drive ribosome assembly.

Acta Cryst. (2020). A76, a76 